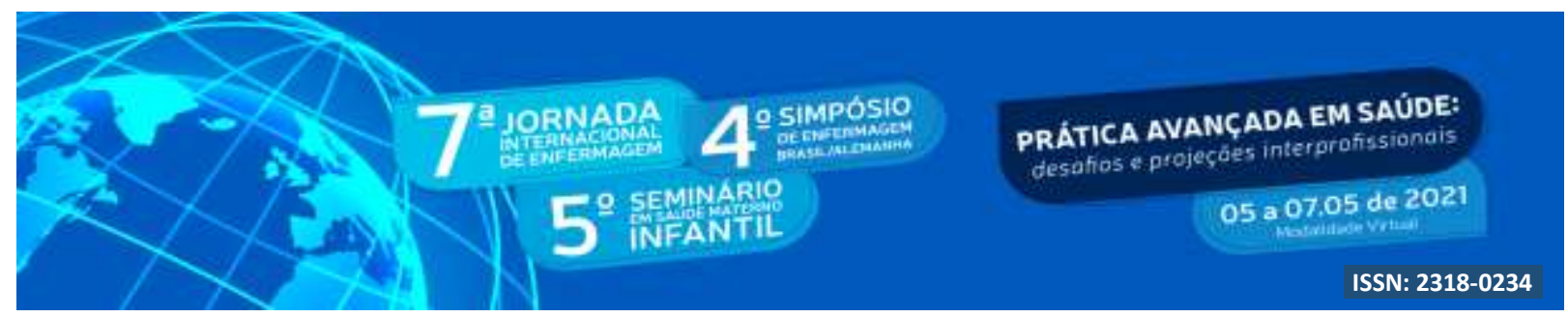

DOI: http://doi.org/10.48195/jie2021-152

\title{
HIPERTENÇÃO ARTERIAL E CONSUMO DE SÓDIO NA POPULAÇÃO DE MILITARES DE SANTA MARIA- RS ${ }^{1}$
}

\author{
Dâniele Giacomelli Tadielo² ${ }^{2}$ Natielen Jacques Schuch ${ }^{3}$
}

\begin{abstract}
RESUMO
O objetivo deste estudo é avaliar a prevalência de Hipertensão Arterial e o consumo alimentar de sódio nos Militares de Santa Maria RS. Trata-se de um estudo transversal, com amostra de 224 militares. Para a análise da HAS, foram considerados níveis elevados a partir de $130 \mathrm{mmHg}$ para pressão sistólica e de $85 \mathrm{mmHg}$ para diastólica de acordo com a NCEP- ATP III, e da IV Diretriz, além de HAS autorreferida. O consumo alimentar foi averiguado pela aplicação de um R24h. A prevalência de HAS foi de $16,1 \%$, e não identificamos associação entre e HAS e consumo de sódio $(\mathrm{p}=0,525)$. Ao analisarmos a mesma associação pela IV Diretriz, também não encontramos associação $(p=0,253)$. Observa-se uma inexistência de associação entre a prevalência de hipertensão arterial e consumo de sódio, provavelmente devido a aplicação de apenas um R24h, sendo considerado uma limitação, já que não representa a ingestão habitual de um indivíduo.
\end{abstract}

Palavras-chave: Exército Brasileiro; Pressão Arterial; Sal.

\begin{abstract}
The aim of this study is to evaluate the prevalence of Arterial Hypertension and sodium food intake in the Military Personnel of Santa Maria RS. This is a cross-sectional study, with a sample of 224 military personnel. For the analysis of SAH, elevated levels were considered starting from $130 \mathrm{mmHg}$ for systolic pressure and $85 \mathrm{mmHg}$ for diastolic according to the NCEP-ATP III, and the IV Guideline, in addition to self-reported SAH. Food consumption was assessed by applying an R24h. The prevalence of SAH was $16.1 \%$, and we did not identify an association between SAH and sodium consumption $(\mathrm{p}=0.525)$. When analyzing the same association through the IV Guideline, we also found no association $(\mathrm{p}=0.253)$. There is no association between the prevalence of arterial hypertension and sodium consumption, probably due to the application of only one R24h, which is considered a limitation, since it does not represent an individual's usual intake.
\end{abstract}

Key Words: Brazilian army; Blood pressure; Salt.

\footnotetext{
${ }^{1}$ Trabalho completo

${ }^{2}$ Estudante do Curso de Mestrado em ciências da Saúde e da Vida. Universidade Franciscana. E-mail: tadielodaniele@gmail.com

${ }^{3}$ Orientadora. Professora do Curso de Nutrição. Universidade Franciscana. E-mail: natielen@yahoo.com.br
} 


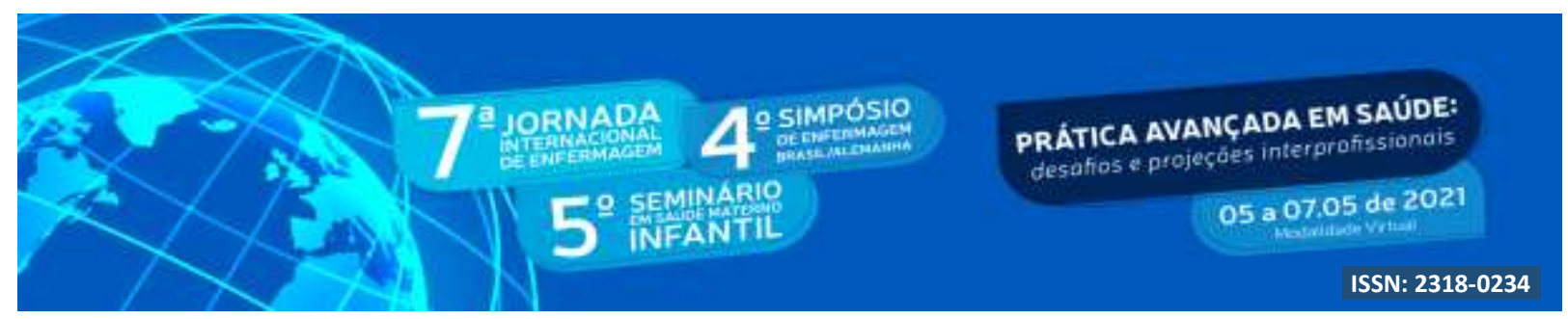

\section{INTRODUÇÃO}

A Organização Mundial da Saúde (OMS) é clara ao afirmar que as doenças crônicas não transmissíveis (DCNT) são consideradas o principal problema de saúde em todo o mundo, causando repercussões negativas na saúde e na qualidade de vida populacional, estando relacionadas às principais causas de morbimortalidade prematura, sendo responsável por mais de $70 \%$ das mortes globais, estando entre as mais frequentes as doenças cardiovasculares, que incluem a hipertensão arterial sistêmica (HAS). Esses agravos possuem uma etiologia complexa, normalmente associada a variáveis comportamentais, sociais, econômicas e demográficas (WORLD HEALTH ORGANIZATION, 2011; 2014; 2017).

A hipertensão arterial sistêmica (HAS) é uma doença crônica e multifatorial em que há elevação sustentada dos níveis pressóricos para valores maiores ou iguais a $140 \mathrm{mmHg}$ para a pressão arterial sistólica (PAS) e/ou $90 \mathrm{mmHg}$ para a pressão arterial diastólica. Possui relevância mundial, estando amplamente associada com o aumento do risco complicações, como infarto agudo do miocárdio, insuficiência cardíaca, doença arterial periférica, acidente vascular encefálico e doença renal crônica (BARROSO et al., 2021; WORLD HEALTH ORGANIZATION, 2014).

No contexto global, identificar e acompanhar os indicadores relacionados às DCNT, incluindo as metas de redução de HAS, é essencial. Assim, sendo necessárias ações para a redução na prevalência de pressão sanguínea elevada, que incluem modificações no padrão alimentar, bem como a redução do consumo de sal, além de esforços para redução do sobrepeso/obesidade e rastreamento para detecção e tratamento prévio das pessoas hipertensas (MALTA et al., 2018).

Estudos apontam elevado consumo de sal em todo o país, englobando todas as faixas etárias e níveis de escolaridade da população brasileira, sendo necessárias intervenções para seu enfrentamento, incluindo, avanço no acesso a alimentos saudáveis por todas as parcelas da população, além de ações para a promoção de educação em saúde, e também estratégias de regulação e monitoramento da indústria alimentícia (MILL et al., 2019; MILL et al., 2021).

Levando em consideração que a hipertensão tem potencial causador de uma média de nove milhões de mortes na população mundial, anualmente, a redução do consumo de sal tem 


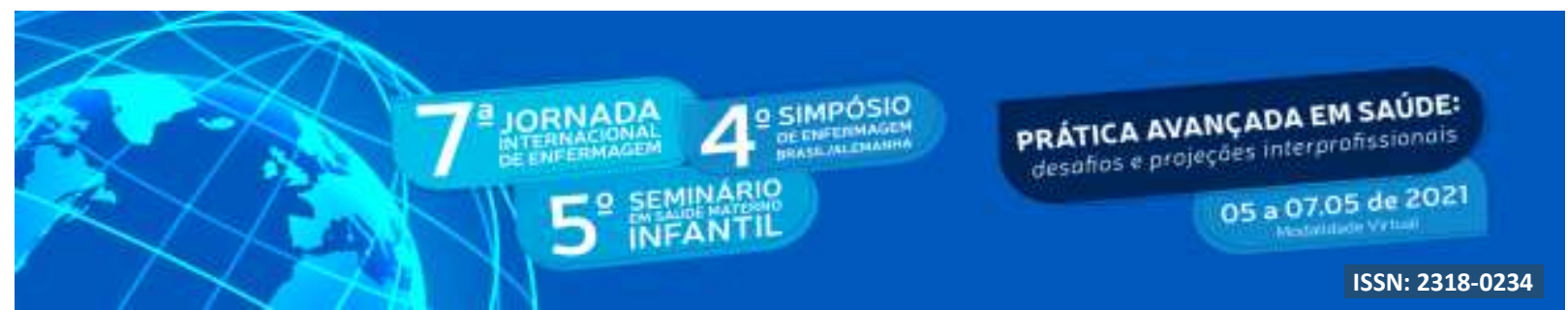

capacidade de redução nos níveis de mortalidade. Por isso, a diminuição no consumo de sal tem sido apontada como uma das ações, não medicamentosas, mais eficazes e de melhor custo no combate das doenças crônicas não transmissíveis, principalmente as doenças cardiovasculares, pela diminuição nos valores médios da pressão arterial na população (MILL et al., 2019). Assim, uma das metas propostas pelo Plano de Ações Estratégicas para o Enfrentamento das DCNT no Brasil (2011-2022) foi a redução no consumo de sal (MALTA; MORAES; SILVA, 2011).

Portanto, a ausência de estudos que abordam o consumo de sódio relacionado à hipertensão arterial de militares da região sul do Brasil, levou a investigação da situação atual dos mesmos. Onde há necessidade de maiores análises a esse respeito, cuja relação com a HAS possa auxiliar no seu impacto nos valores pressóricos.

\section{OBJETIVO}

Avaliar a prevalência de Hipertensão Arterial e a sua relação ao consumo alimentar de sódio dos Militares do Exército Brasileiro da Guarnição de Santa Maria- RS.

\section{METODOLOGIA}

Trata-se de um estudo transversal de caráter quantitativo, descritivo - exploratório, com amostra composta por militares, de ambos os sexos, do $29^{\circ}$ Batalhão de Infantaria Blindada, $4^{\mathrm{a}}$ Batalhão Logístico e do $1^{\circ}$ Regimento de Carros de Combate, das Organizações Militares do Exército Brasileiro do Rio Grande do Sul, de Santa Maria/RS.

Foi utilizada uma amostra não probabilística por conveniência, porém, para reduzir possíveis vieses, totalizando 224 militares, no período de junho a novembro de 2019.

O consumo alimentar de energia, carboidratos, proteínas, lipídeos e sódio, foram averiguados por meio da aplicação de um recordatório alimentar de 24 horas (R24h) aplicado no momento da coleta de dados nos quarteis. As informações obtidas pelo R24h foram computadas utilizando o Programa de Avaliação Nutricional (AVANUTRI) e posteriormente, os dados foram transferidos para uma planilha eletrônica no Excel 2013. 


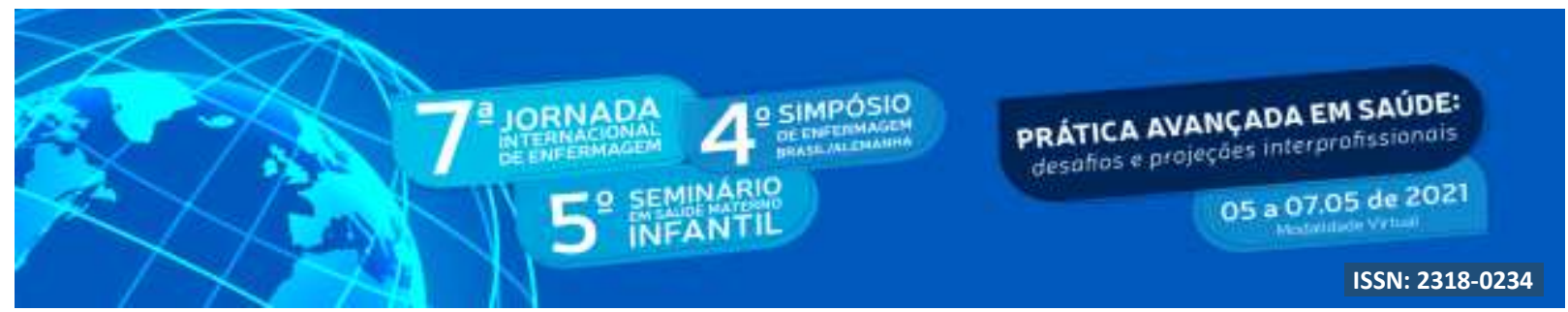

A pressão arterial (PA) foi verificada com a utilização de esfigmomanômetro analógico, marca Becton Dickinson do Brasil@ (São Paulo, Brasil), calibrado pelo INMETRO, de acordo com as recomendações pertinentes. As pressões sistólicas e diastólicas foram obtidas com a média aritmética de duas medidas realizadas no braço esquerdo após o descanso de 5 minutos, enquanto o paciente estava sentado. Para a análise da hipertensão arterial sistêmica (HAS), foram considerados níveis elevados aqueles a partir de $130 \mathrm{mmHg}$ para pressão sistólica e ou de $85 \mathrm{mmHg}$ para pressão diastólica de acordo com os critérios utilizados pelo NCEP- ATP III e da IV Diretriz Brasileira de Hipertensão Arterial, além de indivíduos com HAS autorreferida (GRUNDY et al., 2005; SOCIEDADE BRASILEIRA DE CARDIOLOGIA, 2004).

As análises foram realizadas utilizando-se a versão 21.0 do SPSS (Statistical Package for Social Science Inc., Chicago, Illinois USA). A normalidade da distribuição dos dados numéricos foi verificada por meio do Teste de Kolmogorov-Smirnov. Variáveis quantitativas foram descritas por média e desvio-padrão ou mediana e intervalo interquartil, de acordo com a normalidade ou não de sua distribuição. Foram utilizados os testes Qui-quadrado de Pearson para avaliar a associação entre variáveis categóricas e Teste T de Student, para comparar as medidas quantitativas.

Este trabalho foi aprovado pelo Comitê de Ética em Pesquisa da Universidade Franciscana, pelo parecer $n^{\circ}$ 3.193.459., ficando registrado no CAAE 0817019 .0.0000.5306, e segue as diretrizes e normas que regulamentam as pesquisas envolvendo seres humanos, aprovadas pela Resolução $\mathrm{n}^{\circ}$ 422, de 22 de dezembro de 2012, do Conselho Nacional de Saúde e, uma vez aprovado, os participantes assinaram o Termo de Consentimento Livre e Esclarecido (TCLE), sendo entregue em duas vias, com o Termo de Confidencialidade de pesquisa. Salienta-se que essa pesquisa obteve parecer favorável pela Comissão de Ética Médica do Hospital Geral de Santa Maria (HGeSM).

\section{RESULTADOS E DISCUSSÃO}

Foram investigados 224 militares, a maioria do gênero masculino $(94,6)$ com média de

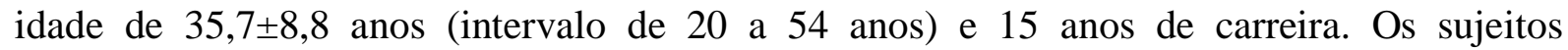




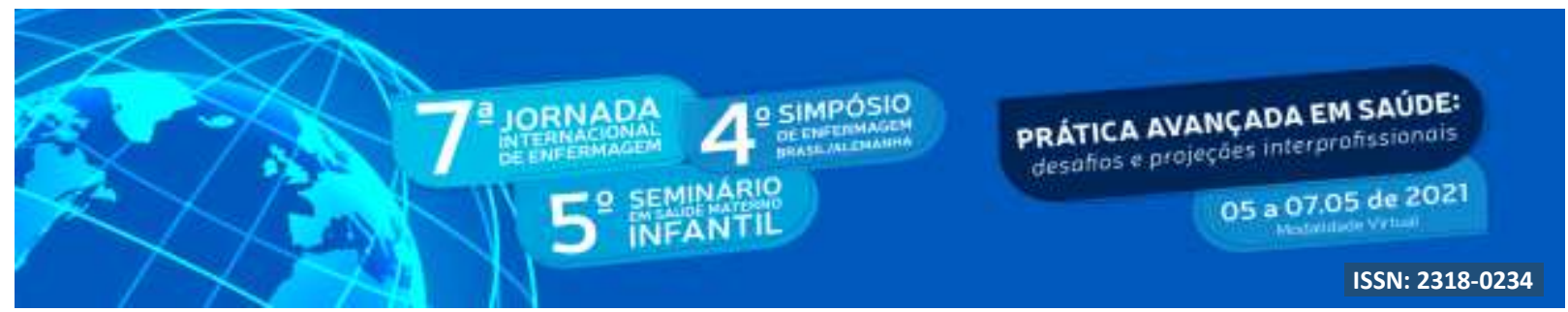

apresentaram peso médio de $80,8 \mathrm{~kg}$, IMC médio de $26,7 \mathrm{~kg} / \mathrm{m} 2$, sendo $(67,4 \%)$ classificados com sobrepeso ou obesidade e $(95,1 \%)$ praticantes de atividade física.

A prevalência de HAS foi de $16,1 \%$ na população estudada, onde os participantes com HAS eram mais velhos $(\mathrm{P}<0,001)$. No Brasil, os dados de prevalência tendem a mudar de acordo com a metodologia utilizada. Segundo a Pesquisa Nacional de Saúde de 2013, 21,4\% dos adultos brasileiros autorrelataram HAS, em contrapartida, levando em consideração a aferição da PA e uso de medicamentos anti-hipertensivos, o percentual de adultos com HAS chegou a 32,3\%. Identificou-se, que essa prevalência foi superior entre homens, além de, aumentar com o envelhecimento por todos os critérios, chegando a 71,7\% para os indivíduos com idade maior que 70 anos (MALTA et al., 2018).

Com o envelhecimento, o aumento dos níveis pressóricos, torna-se uma complicação significativa, onde cerca de $65 \%$ da população com idade superior a 60 anos manifestam quadro de HAS. Também, deve-se, levar em consideração o fato de Brasil estar passando por uma transição epidemiológica, com um número ainda maior de idosos ( $\geq 60$ anos) nas próximas décadas, provocando um crescimento da prevalência de HAS, bem como das complicações causadas pela mesma (MENNI et al., 2013).

Em relação à avaliação antropométrica, militares com HAS apresentavam peso e IMC mais elevados, sendo classificados, mais frequentemente, como obesos $(\mathrm{P}<0,001)$. Observa-se uma associação direta, contínua e quase linear entre o excesso de peso e os níveis de pressão arterial. Onde, tanto a obesidade geral, quanto a abdominal foram relacionadas ao aumento do risco de HAS. No entanto, uma diminuição no peso provoca a diminuição da pressão arterial em sujeitos hipertensos, e também em normotensos (PRÉCOMA et al., 2019; CAREY et al., 2018; WHELTON et al., 2017).

Considerando a associação entre HAS e gênero, encontramos associação $(p=0,000)$ tanto pelo critério do NCEP ATPIII quanto pelo da IV Diretriz Brasileira de Hipertensão Arterial, onde a prevalência de hipertensão é maior entre os indivíduos do gênero masculino. Resultado este, que pode ser explicado pelo fato de a população estudada ser de militares, que em sua maioria é composta por indivíduos do gênero masculino. Pois, segundo Menni et al., (2013), a PA é mais elevada entre homens, porém em faixas etárias mais jovens. Já na população com 60 anos de idade, os níveis pressóricos apresentam-se mais elevados nas 


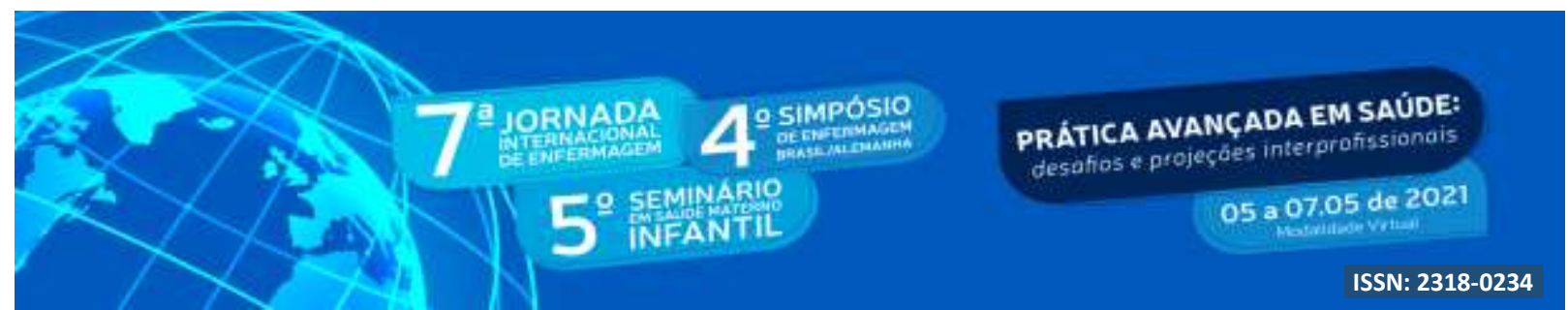

mulheres, sendo maior a prevalência de HAS. Em ambos os sexos, a frequência de HAS aumenta com a idade, alcançando 61,5\% e 68,0\% na faixa etária de 65 anos ou mais, em homens e mulheres, respectivamente.

Segundo a classificação do NCEP ATP III não encontramos associação entre e HAS e consumo de sódio $(\mathrm{p}=0,525)$. Ao analisarmos a mesma associação entre HAS e a IV Diretriz Brasileira de Hipertensão Arterial, também não encontramos associação ( $p=0,253)$. Sendo importante ressaltar que a aplicação do recordatório alimentar 24 horas, com a população estudada, foi realizada em apenas um dia, o que potencialmente poderia ser considerado uma limitação, já que não representa a ingestão habitual de um indivíduo. Essa limitação se deve à elevada variabilidade do consumo de nutrientes intrapessoal e interpessoal, o que confere ao método pouca representatividade do consumo alimentar habitual.

Tabela 1. Variáveis de consumo, segundo a presença ou não de Hipertensão Arterial na população de militares de Santa Maria - RS (N=224).

\begin{tabular}{|c|c|c|c|c|}
\hline \multirow[b]{2}{*}{ Variável } & \multirow[b]{2}{*}{ Recomendação* } & \multicolumn{2}{|c|}{ Hipertensão Arterial } & \multirow[b]{2}{*}{$\mathbf{P} * *$} \\
\hline & & 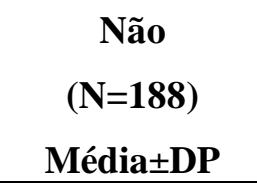 & $\begin{array}{c}\text { Sim } \\
(\mathrm{N}=36) \\
\text { Média } \pm \mathrm{DP}\end{array}$ & \\
\hline$\%$ Carboidratos & $45-65$ & $50.4 \pm 12.4$ & $50.6 \pm 11.8$ & 0,924 \\
\hline \% Lipídeos & $20-35$ & $29.5 \pm 7.9$ & $29.3 \pm 8.2$ & 0,833 \\
\hline$\%$ Proteínas & $10-35$ & $19.9 \pm 7.2$ & $20.0 \pm 5.7$ & 0,936 \\
\hline Valor Calórico Total (Kcal) & 2000 & $1935.3 \pm 771.0$ & $2010.3 \pm 724.4$ & 0,455 \\
\hline Sódio (mg) & 2300 & $2508.1 \pm 1674.1$ & $2654.7 \pm 1832.6$ & 0,528 \\
\hline
\end{tabular}

*Fonte: Institute of Medicine

**Teste T de Student

\section{CONCLUSÃO}

Em comparação aos demais estudos, que abrangem a população brasileira, os nossos resultados, mostraram menor prevalência de hipertensão arterial entre os militares estudados. Também, observa-se pelos resultados do estudo, em oposto aos demais já existentes, uma inexistência de associação entre a prevalência de hipertensão arterial e consumo de sódio. 


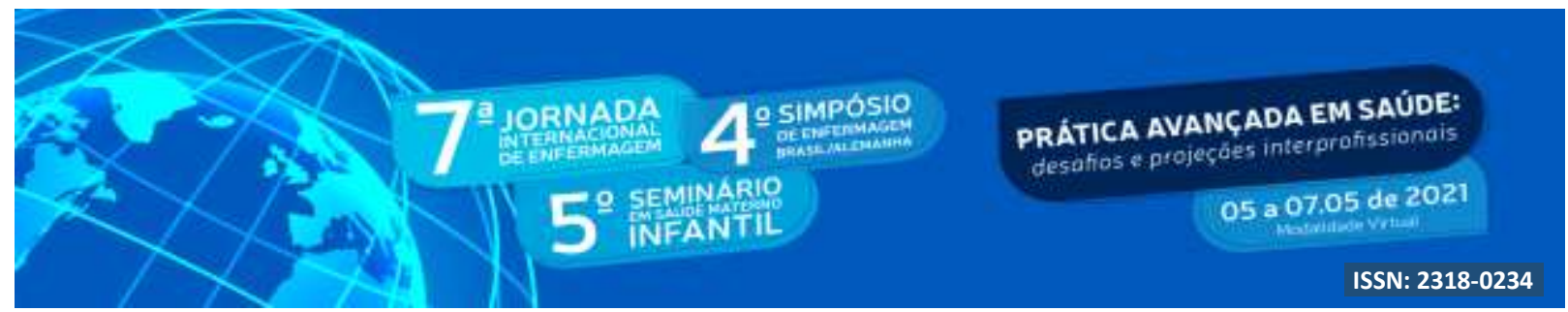

Onde, a dificuldade para se estimar as quantidades de sal acrescentadas no preparo dos alimentos, é considerado um grande viés do estudo.

Ainda assim, deve-se estimular o planejamento para ações de controle da HAS, destacando a importâcia de um padrão alimentar equilibrado e saudável, já que à longo prazo, mudanças alimentares levam a redução de valores antropométricos, bem como dos níveis pressóricos.

\section{REFERÊNCIAS}

BARROSO, W. K. S. ET AL. Diretrizes Brasileiras de Hipertensão Arterial - 2020. São Paulo: Arquivo Brasileiro de Cardiologia, 2021.

CAREY, R. M. ET AL. Prevention and Control of Hypertension: JACC Health Promotion Series. Journal of the American College of Cardiology, 2018.

MALTA, D. C. ET AL. Prevalência da hipertensão arterial segundo diferentes critérios diagnósticos, Pesquisa Nacional de Saúde. São Paulo: Revista Brasileira de Epidemiol, 2018.

MALTA, D. C; MORAES, O. L; SILVA, J. B. Apresentação do plano de ações estratégicas para o enfrentamento das doenças crônicas não transmissíveis no Brasil, 2011 a 2022. Brasília: Epidemiolia Serviço Saúde, 2011.

MENNI, C. ET AL. Heritability analyses show visit-to-visit blood pressure variability reflects different pathological phenotypes in younger and older adults: evidence from UK twins. Journal of Hypertension, 2013.

MILL, J. G. ET AL. Estimativa do consumo de sal pela população brasileira: resultado da Pesquisa Nacional de Saúde 2013. São Paulo: Revista Brasileira de Epidemiologia, 2019.

MILL, J. G. ET AL. Fatores associados ao consumo de sal na população adulta brasileira: Pesquisa Nacional de Saúde. São Paulo: Revista Ciência e Saúde Coletiva, 2021.

PRÉCOMA, D. B. ET AL. Atualização da Diretriz de Prevenção Cardiovascular da Sociedade Brasileira de Cardiologia - 2019. Arquivo Brasileiro de Cardiologia, 2019. Disponível em: https://abccardiol.org/article/atualizacao-da-diretriz-de-prevencaocardiovascular-da-sociedadebrasileira-de-cardiologia-2019/

SOCIEDADE BRASILEIRA DE CARDIOLOGIA - SBC. IV Diretrizes Brasileiras de Hipertensão Arterial. Arquivo Brasileiro de Cardiologia, 2004. Disponível em: https://www.scielo.br/scielo.php?script=sci_arttext\&pid=S0066-782X2004001000004 


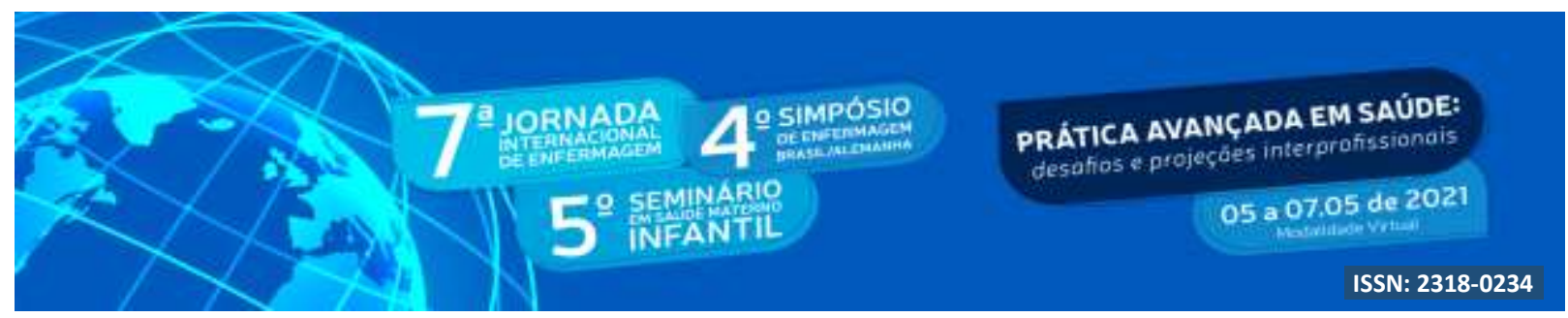

WHELTON, P. K. ET AL. 2017 Guideline for Prevention, Detection, Evaluation and Management of High Blood Pressure in Adults. Journal of the American College of Cardiology, 2017. Disponível em: https://www.jacc.org/doi/full/10.1016/j.jacc.2017.11.006

WORLD HEALTH ORGANIZATION - WHO. Global status report on noncommunicable diseases 2014. Geneva: World Health Organization, 2014. Disponível em:

https://www.who.int/nmh/publications/ncd-status-report-2014/en / 